\title{
CAPSULE COMMENTARIES \\ Capsule Commentary on Ganguli et al., What Do High-Risk Patients Value? Perspectives on a Care Management Program
}

\author{
Sierra Tackett, BS and Jeffrey L. Jackson, MD MPH
}

Medical College of Wisconsin, Milwaukee, WI, USA.

$\mathrm{J}$ Gen Intern Med 33(1):87

DOI: $10.1007 / \mathrm{s} 11606-017-4220-\mathrm{x}$

() Society of General Internal Medicine 2017

$\mathrm{G}$ anguli et al. surveyed high-risk patients enrolled in a care coordination management program in order to obtain their perspectives on the program. ${ }^{1}$ Many institutions are implementing programs for high-risk patients; most are motivated by a desire to reduce utilization and cost and improve patient outcomes. Such interventions demonstrate variable success. ${ }^{2}$ While Ganguli's program is one of the few demonstrating reduced utilization and costs, the authors posit that other outcomes, such as satisfaction, quality of life, and perceived care integration, are important and more realistic targets for these programs. They found that participants in this program reported helpful interactions with their care team around medical and social determinants of health.

Other studies on interventions to improve high-risk patient care have generally lacked the patient's perspective. ${ }^{3}$ It is unclear from this study which aspects of the program patients found particularly valuable. It is perhaps not surprising that those patients with greater worry may interact with the health care system more frequently, including interactions with their care coordinator. Determining which subgroups might be more responsive to targeted care coordination could be helpful to policymakers. Ganguli's findings, though limited, suggest that women, younger patients, those with greater health worry, those with a greater sense of health control, and those who prefer to defer health decisions to their provider are more likely to recall interactions with the coordination program than are other participants.
While greater health control may result in more active participation in healthy behaviors, ${ }^{4}$ being passive has generally been associated with a reduced sense of health control, so this latter finding is paradoxical. What is lacking from these data are other potential markers for subgroups that may be responsive to a care coordination program, such as cognition, somatization, mental disorders, literacy, numeracy and functional status. It would also be helpful to determine whether the patients in this study who recalled helpful interactions with the health care team were also those who experienced cost savings. Unfortunately, there are many patient-specific characteristics that cause patients to be resource-intensive, and not all may prove amenable to improvement through care coordination. Studies to identify these subgroups are needed.

Corresponding Author: Sierra Tackett, BS; Medical College of Wisconsin, Milwaukee, WI, USA (e-mail: Stackett@mcw.edu).

\section{Compliance with Ethical Standards:}

Conflict of Interest: The authors have no conflict of interest with this article.

\section{REFERENCES}

1. Ganguli I, Orav EJ, Weil E, Gerris TG, Vogeli C. What do high-risk patients value? Perspectives on a care management program. J Gen Intern Med. https://doi.org/10.1007/s11606-017-4200-1.

2. Bodenheimer T, Berry-Millett R. Follow the money-controlling expenditures by improving care for patients needing costly services. N Engl $\mathrm{J}$ Med 2009;361:1521-3.

3. Peikes D, Chen A, Schore J. Effects of care coordination on hospitalization, quality of care and health care expenditures among Medicare beneficiaries, 15 randomized trials. JAMA. 2009; 301(6):603-618.

4. Menec VH. Chipperfield JG, Perry RP. Self-perceptions of health: a prospective analysis of mortality, control and health. J Gerontol 1999;54(2):85-93. 